\title{
Tipos de colostomía según su localización y la calidad de vida en los pacientes colostomizados que asisten al Consultorio Externo de Cirugía General del Hospital Nacional Guillermo Almenara Irigoyen, Lima, 2015
}

\author{
Types of colostomy according to location and life quality in patients attending to Outpatient of \\ General Surgery of the Guillermo Almenara Irigoyen, National Hospital, Lima, 2015
}

Jairo Bastidas Chuquillanqui ${ }^{*}$, Jhoselyn Galarza Ríos², Wilmer Calsin Pacompia ${ }^{3}$

\begin{abstract}
RESUMEN
Objetivo: Determinar la relación entre los tipos de colostomía según su localización y la calidad de vida en los pacientes colostomizados. Material y métodos: estudio de enfoque cuantitativo, diseño no experimental, de tipo correlacional, transversal. El tipo de muestreo fue no probabilístico siendo por conveniencia bajo criterios de inclusión y exclusión. La muestra estuvo constituida por 90 pacientes colostomizados. Se usó el Cuestionario SF36 para medir la calidad de vida y para el tipo de colostomía según su localización, se utilizó una guía de evaluación. Resultados: Se evidenció que existe relación entre colostomía ascendente, transverso y calidad de vida en la dimensión física y mental con $\mathrm{p}=0,031$ y 0,011 y p=0,008 y 0,007, respectivamente. En cuanto a colostomía descendente y calidad de vida en la dimensión física no existe relación $(p=0,286)$ y en la dimensión mental existe relación ( $\mathrm{p}=0,031$ ), asimismo, en la colostomía sigmoidea y calidad de vida en las dimensiones física y mental no existe relación ( $\mathrm{p}=0,210$ y 0,340). Existe relación entre el tiempo de permanencia de la colostomía y la calidad de vida $(\mathrm{p}=0,036)$. La calidad de vida global de los pacientes colostomizados es de nivel bajo 27,8\%, medio 51,5\% y alto 13,4\%. Conclusión: los tipos de colostomía según su ubicación y la calidad de vida de los pacientes colostomizados se relacionan significativamente ( $\mathrm{p}$ value $=0,028$ ), ya que estos pacientes experimentan situaciones desagradables debido a los cambios fisiológicos, dietéticos, psicológicos, estéticos, sociales y económicos que experimentan tras la cirugía.
\end{abstract}

Palabras Clave: Paciente colostomizado, calidad de vida, colostomía.

\begin{abstract}
Objective: Determine the relationship between the types of colostomy according to location and life quality in colostomy patients. Methodology: Quantitative approach study, non-experimental design, correlational, cross type. The type of sampling was non probabilistic by convenience under inclusion and exclusion criteria. The sample consisted by 90 colostomy patients. The SF36 questionnaire was used to measure the quality of life and the type of colostomy according to location, an evaluation guide was used. Results: It was evident That a relationship exists Between colostomy ascending , transverse and quality of life in physical and mental dimension With 0.031 and $p=0.011$ and $p=0.008$ and 0.007 , respectively. Once a descending colostomy and quality of life in the physical dimension There is no relationship $(\mathrm{p}=0.286)$ and there mental dimension ratio $(\mathrm{p}=0.031)$ Also in the sigmoid colostomy and Quality of Life in the physical dimensions of mind and there is no relationship $(\mathrm{p}=0.210$ and 0.340$)$. There relationship Between time spent colostomy and quality of life $(\mathrm{p}=0.036)$. Social Life Quality of Patients colostomy is Low Level 27.8 \% 51.5 \% 13.4 \% Medium and High. Conclusion: the types of colostomy according to location and the quality of life of colostomy patients are related significantly ( $\mathrm{p}$ value $=0,028$ ), because these patients experience unpleasant situations due to physiological, dietary, psychological, aesthetic, social and economic changes.
\end{abstract}

Keywords: Colostomy patient, quality of life, colostomy.

${ }^{1}$ Licenciado en Enfermería. Clínica Angloamericana, San Isidro, Perú

${ }^{2}$ Licenciada en Enfermería, Clínica Good Hope, Miraflores, Lima, Perú

${ }^{3}$ Docente, EP Enfermería, Universidad Privada Norbert Wiener, Lima, Perú 
Tipos de colostomía según su localización y la calidad de vida en los pacientes colostomizados que asisten al Consultorio Externo de Cirugía General del Hospital Nacional Guillermo Almenara Irigoyen, Lima, 2015

\section{INTRODUCCIÓN}

El concepto de calidad de vida ha cobrado importancia en los últimos tiempos ya que, al aumentar la expectativa de vida en la población, también se ha incrementado el número de personas con algún grado de discapacidad o con enfermedades crónicas, que sufren los efectos propios de la enfermedad o del tratamiento médico o quirúrgico (Organización Mundial de la Salud, 2015).

Y mas aun, la calidad de vida es un estado de satisfacción general, derivado de la realización de las potencialidades de la persona. Posee aspectos subjetivos y objetivos. Es una sensación subjetiva de bienestar físico, psicológico y social. Incluye como aspectos subjetivos la intimidad, la expresión emocional, la seguridad percibida, la productividad personal y la salud percibida. Como aspectos objetivos el bienestar material, las relaciones armónicas con el ambiente (físico, social y comunidad) y la salud objetivamente considerada (Ardila, 2003)

Por otro lado, la colostomía como la creación quirúrgica de una abertura del colon a la piel de la pared abdominal y su propósito es desviar el tránsito intestinal o descomprimir el intestino abdominal, es un tipo de operación requerida cuando una persona ha perdido las funciones del intestino grueso, este tipo de estomas permitirá que los desperdicios normales del cuerpo sean expedidos por una abertura en la pared abdominal (Villajuan, 2006).

La técnica de una colostomía es el tratamiento más adecuado desde el punto de vista quirúrgico, sin embargo ocurren complicaciones postoperatorias y cambios fisiológicos, psicológicos y sociales en el paciente, que afectan directamente a su calidad de vida, ya que al realizar un estoma de eliminación, implica además de una alteración de la imagen corporal; la pérdida del control voluntario de eliminación y la necesidad de utilizar una bolsa para almacenar las materias fecales vertidas por el intestino (García et al., 2014).

En consecuencia, el estudio de la calidad de vida de los pacientes colostomizados se ha convertido en una ayuda vital para el personal del área de la salud, ya que a través de ésta se pueden detectar fallas en la forma de proceder del personal médico o enfermero, que atiende al paciente, además, se logra determinar si lo que se hace es suficiente o no, y así diseñar cada vez mejores métodos para ayudar al paciente. (Ferreira-Umpiérrez, 2013)

Es así que para el profesional de enfermería estudiar la calidad de vida en los pacientes colostomizados es de gran interés, porque permite conocer al paciente desde una perspectiva más holística, que incluye sus valores, creencias, percepciones, etc, por ende tal conocimiento permitirá al enfermero (a) a canalizar sus cuidados de acuerdo a la patología, estado de salud y características personales y con ello mejorar la calidad de vida del paciente a cargo (Castro, M., Castro, C., \& Clemente, H., 2013).

Por ende el objetivo del trabajo de investigacion es determinar la relación entre los tipos de colostomía según su localización y la calidad de vida en los pacientes colostomizados, que asisten al Consultorio Externo de Cirugía General del Hospital Nacional Guillermo Almenara Irigoyen, Lima.

\section{MATERIALES Y MÉTODOS}

El estudio es de enfoque cuantitativo, diseño no experimental, de tipo correlacional, transversal. El tipo de muestreo fue no probabilístico siendo por conveniencia bajo criterios de inclusión y exclusión. La muestra estuvo constituida por 90 pacientes colostomizados. Se usó el Cuestionario SF36 para medir la calidad de vida y para el tipo de colostomía según su localización, se utilizó una guía de evaluación.

Para la recolección de datos se utilizó dos instrumentos, para determinar el nivel de calidad de vida en los pacientes colostomizados se utilizó el cuestionario de salud SF-36, el cual consta de 36 temas, que exploran 8 dimensiones del estado de salud: función física (10 ítems); función social (4 ítems), dolor corporal (2 ítems); y salud general (5 ítems); que son parte del componente de salud física. Así mismo vitalidad (4 ítems); función social (2 ítems); rol emocional (3 ítems); y salud mental (5 ítems); que son parte del componente de salud mental. Adicionalmente un ítem que es de transición de salud, el cual no se utiliza para ningún cálculo de ninguna de las escala, pero es útil para comprender el cambio percibido del estado de salud.

Por otro lado para determinar el tipo de colostomía se utilizó guía estructurada por los investigadores, la cual fue validado por juicio de expertos, la cual consta, de preguntas generales y una imagen en donde el paciente ubicará el lugar de su colostomía, luego los investigadores determinaron la ubicación basándose en la historia clínica.

Para recolección de datos se coordina con el Hospital 
Guillermo Almenara Irigoyen y la facultad de ciencias de la salud para los permisos respectivos asimismo se coordinó con jefatura de enfermería para determinar la hora y fecha para la aplicación del instrumento, se desarrolló en el mes Diciembre en diferentes turnos.
Para el procesamiento y análisis de datos se utilizó el paquete estadístico SPSS v20, y para identificar la relación de variables se utilizó la estadística inferencial de Chi cuadrado.

\section{RESULTADOS}

Tabla 1

Tipos de colostomía según su localización y la calidad de vida de los pacientes que asisten a consultorio externo de Cirugía General del HNGAI, 2015

\begin{tabular}{|c|c|c|c|c|c|c|c|c|c|c|}
\hline \multicolumn{11}{|c|}{ Tipos de colostomía según su localización } \\
\hline \multirow{2}{*}{$\begin{array}{l}\text { Calidad de } \\
\text { vida } \\
\text { Baja }\end{array}$} & \multicolumn{2}{|c|}{$\begin{array}{l}\text { Ascendente } \\
\mathrm{n} \%\end{array}$} & \multicolumn{2}{|c|}{$\begin{array}{l}\text { Transversa } \\
\mathrm{n} \%\end{array}$} & \multicolumn{2}{|c|}{$\begin{array}{l}\text { Descendente } \\
\mathrm{n} \%\end{array}$} & \multicolumn{2}{|c|}{$\begin{array}{l}\text { Sigmoidea } \\
\mathrm{n} \%\end{array}$} & \multicolumn{2}{|c|}{$\begin{array}{l}\text { Total } \\
\mathrm{n} \%\end{array}$} \\
\hline & 4 & $4,4 \%$ & 11 & $12,2 \%$ & 7 & $7,8 \%$ & 5 & $5,6 \%$ & 27 & $30,0 \%$ \\
\hline Media & 18 & $20,0 \%$ & 5 & $5,6 \%$ & 18 & $20,0 \%$ & 9 & $10,0 \%$ & 50 & $55,6 \%$ \\
\hline Alta & 2 & $2,2 \%$ & 2 & $2,2 \%$ & 7 & $7,8 \%$ & 2 & $2,2 \%$ & 13 & $14,4 \%$ \\
\hline
\end{tabular}

En la tabla 1 se evidencia un nivel de calidad de vida baja en los pacientes con colostomía transversa (12,2\%), descendente (7,8\%), sigmoidea (5,6\%) y un porcentaje menor en quienes tienen colostomía de tipo ascendente $(4,4 \%)$, en tanto, se observa un nivel de calidad de vida media en los tipos de ascendente
(20\%) ,descendente (20\%), sigmoidea (10\%) y de tipo transversa $(5,6 \%)$, asimismo, los pacientes con colostomía de tipo descendente transversa ascendente y sigmoidea tienen un nivel de calidad de vida alta con $7,8 \%, 2,2 \%, 2,2 \%$ y $2,2 \%$, respectivamente.

Tabla 2

Relación entre tipos de colostomía según su localización y calidad de vida

\begin{tabular}{llll}
\hline & Valor & Gl & Sig. Asintótica \\
\hline Chi-cuadrado de Pearson & $14,104^{\mathrm{a}}$ & 6 & 0,028 \\
\hline
\end{tabular}

Se observa que en la Tabla 2, el valor de $\mathrm{Chi}^{2}$ que es igual a 0,028 , ( $p$ value $<0,05$ ), por consiguiente se rechaza la hipótesis nula lo que significa que existe una relación significativa entre los tipos de colostomía según la localización y la calidad de vida de los pacientes colostomizados.

Tabla 3

Relación entre el tipo de colostomía y calidad de vida en la dimensión física

\begin{tabular}{llll}
\hline Tipo de colostomía & Valor Chi-cuadrado de Pearson & Gl & Sig. Asintótica \\
\hline Ascendente & $2,338^{\mathrm{a}}$ & 2 &, 031 \\
Transversa & $9,692^{\mathrm{a}}$ & 2 &, 008 \\
Descendente & $2,501^{\mathrm{a}}$ & 2 &, 286 \\
Sigmoidea &, $347^{\mathrm{a}}$ & 2 &, 340 \\
\hline
\end{tabular}

En la Tabla 3, se muestra que en los tipos de colostomía ascendente y transversa y la calidad de vida en la dimensión física tiene un $p<0,05$, por consiguiente se rechaza en ambos casos la hipótesis nula, lo que significa que existe una relación significativa entre las dos variables, respectivamente. Por otro lado, los respecto a los tipos de colostomía descendente y sigmoidea y calidad de vida en la dimensión física el valor $p>0,05$, por consiguiente se acepta la hipótesis nula, es decir, no hay asociación entre esos tipos de colostomía y dimensión física de calidad de vida. 
Tipos de colostomía según su localización y la calidad de vida en los pacientes colostomizados que asisten al Consultorio Externo de Cirugía General del Hospital Nacional Guillermo Almenara Irigoyen, Lima, 2015

Tabla 4

Relación entre el tipo de colostomía y calidad de vida en la dimensión mental

\begin{tabular}{llll}
\hline Tipo de colostomía & Valor Chi-cuadrado de Pearson & Gl & Sig. Asintótica \\
\hline Ascendente & $4,419^{\mathrm{a}}$ & 2 &, 011 \\
Transversa &, $633^{\mathrm{a}}$ & 2 &, 007 \\
Descendente & $3,126^{\mathrm{a}}$ & 2 &, 031 \\
Sigmoidea &, $213^{\mathrm{a}}$ & 2 &, 210 \\
\hline
\end{tabular}

En la Tabla 4, se muestra que en los tipos de colostomía ascendente, transversa y descendente en relación a la calidad de vida en la dimensión mental tiene un $\mathrm{p}<0,05$, lo que significa que existe una relación significativa entre las dos variables. Por otro lado, la colostomía sigmoidea y calidad de vida en la dimensión física el valor $\mathrm{p}$-value $=$ es mayor a 0,05 por consiguiente se acepta la hipótesis nula, es decir, no hay asociación entre dichas variables.

\section{Tabla 5}

Tiempo de permanencia de la colostomía y la calidad de vida de los pacientes que asisten a consultorio externo de cirugía General del HNGAI, 2015

\begin{tabular}{|c|c|c|c|c|c|}
\hline & & \multicolumn{3}{|c|}{ Calidad de vida } & \multirow[t]{2}{*}{ Total } \\
\hline & & Baja & Media & Alta & \\
\hline \multirow{4}{*}{$\begin{array}{l}\text { Tiempo de } \\
\text { permanencia de la } \\
\text { colostomía }\end{array}$} & Más de dos años & 2 & 3 & 15 & 20 \\
\hline & & $10,0 \%$ & $15,0 \%$ & $75,0 \%$ & $100,0 \%$ \\
\hline & De 1 a 2 años & 24 & 35 & 11 & 70 \\
\hline & & $34,3 \%$ & $50,0 \%$ & $15,7 \%$ & $100,0 \%$ \\
\hline
\end{tabular}

p:,036

La Tabla 5, con respecto a los pacientes que portan una colostomía por más de dos años el 75 \% presentó una calidad de vida alta, mientras que el 50\% de los pacientes que portan una colostomía entre uno a dos años presentan una calidad de vida media, es decir, a más tiempo de tener la colostomía existe mayor adaptación y por ende una calidad de vida alta, entre menos tiempo menor adaptación, por tanto, una calidad de vida baja. Asimismo existe una relación significativa entre las dos variables un $p$-value $=036$.

\section{DISCUSIÓN}

El propósito del presente estudio fue determinar la calidad del paciente colostomizado que asiste al consultorio externo de cirugía general .En este parte se analizan a modo de discusión los resultados obtenidos en cada cuestionario aplicado durante el periodo del estudio.

Se valoraron los resultados obtenidos tanto en el análisis cuantitativo de los datos como el análisis de contenido y se exponen los resultados finales respecto a las variables.
Durante siglos, el pensamiento y ejercicio enfermero han sido llevados a cabo desde una perspectiva biomédica. Esto cambió con la incorporación del proceso científico y el desarrollo de un marco conceptual propio de la profesión, lo que a su vez originó el desarrollo de los planes de atención de enfermería y con ello la mejora de los cuidados de la salud de los pacientes, por otro lado, la realización de una colostomía supone un gran impacto en el modo y calidad de vida de los pacientes sometidos a esta intervención, por lo que necesitan una atención especializada desde un punto de vista holístico y humanizado (Crespo, 2011).

El objetivo del estudio fue determinar la relación entre los tipos de colostomía según su localización y la calidad de vida, como resultado se obtuvo un p-value $=0,028$ lo cual indica la relación de ambas variables, esta asociación se explica porque los pacientes colostomizado experimentan situaciones desagradables debido a los cambios fisiológicos (tipo de colostomía), dietéticos, psicológicos, estéticos, sociales y económicos (Espinoza,2003) alterando su nivel de calidad de vida (55,60\% media y 30.00\% baja) es decir, presenta deficiencia en la destreza de 
su propio cuidado, miedo al rechazo por el olor de la bolsa, temor, depresión y ansiedad por el nuevo cambio de su imagen corporal.

Por otro lado, Charúa-Guindic (2011) realizó un estudio la calidad de vida del paciente colostomizado, dando como resultado que $68,7 \%$ presentó una calidad de vida aceptable para los distintos reactivos. La diferencia de estos resultados, se explica porque la percepción de una persona varia en el contexto cultural y de valores inmersos en relación con sus objetivos, normas y oportunidades para la sensación de sentirse bien (OMS, 2015).

En relación a los tipos de colostomía (ascendente y transversa ) y la calidad de vida, en la dimensión física y mental, el resultado para la colostomía ascendente tuvo p-value $=0,031$ y 0,011 , para la colostomía transversa un p-value $=008$ y 007 , en la dimensiones física y mental, respectivamente, estos datos indican una asociación significativa entre estas variables, por lo tanto; esta asociación se debe; a que al modificar el sitio de eliminación de las heces se está afectando también la autopercepción del paciente y por ende su autoestima, que posteriormente se podría derivar a un problema mental (depresión, ansiedad, etc), así mismo una colostomía ascendente o transversa, influye grandemente en la calidad de vida, debido a que el estoma realizado es muy grande y cuenta con dos aberturas (una abertura expulsa la materia fecal; la otra expulsa sólo la mucosidad), el flujo de las heces son constantes y de un olor muy marcado, además, en esta porción de intestino grueso se da la absorción de agua y electrolitos un gran porcentaje (Gonzales, 2009), las complicaciones de tipo periostomal son frecuentes en estos tipos de colostomía, debido que las heces son liquidas y contienen enzimas digestivas que son irritantes para la piel del paciente, así mismo la parte de intestino grueso que está en reposo seguirá produciendo mucosidad y que será eliminado a través del ano (Sánchez, 2014).

Por otro lado, un estudio realizado en Perú por Villajuan (2006) reporta que los pacientes colostomizados están en proceso de recuperación de su calidad de vida, presentando en la dimensión biológica una calidad de vida baja y en la dimensión psicológica una calidad de vida media, sin embargo tal investigación refleja una evaluación global de la calidad de vida del paciente colostomizado, que en cierto modo se asemeja a las variables de estudio, ya que la calidad de vida de los pacientes con colostomía ascendente y transversa, están en proceso de recuperación, debido a que traen consigo un porcentaje elevado de complicaciones y de cuidados específicos que muchas veces los pacientes desconocen (Pita et al., 2015).
Respecto a la relación entre la los tipos de colostomía (descendente y sigmoidea) y la calidad de vida, en la dimensión física y mental, el resultado para la colostomía descendente se obtuvo un p-value $=0,286$ y 0,031 , para la colostomía sigmoidea un p-value $=$ 0,340 y 0,210, en la dimensión física y mental, respectivamente, por otro lado, la calidad de vida en colostomía descendente dio resultado en la dimensión mental que si existe asociación significativa, como también indican que no existe asociación significativa entre las demás variables.

Los datos en donde no existe relación, se debe en cierta manera a la ubicación del estoma, ya que por ser más cercano al ano, existe un bajo riesgo de complicaciones periostomales, debido a que las heces son más consistentes y no contienen enzimas digestivas (Sánchez, 2014), además el movimiento intestinal (para la evacuación) ocurrirá después de que cierta cantidad de materia fecal se haya acumulado en el intestino, teniendo así un control voluntario sobre la evacuación intestinal ya que diversos estudios han demostrado que de 100 pacientes con colostomía, 1/3 hacen 1-2 deposiciones al día, y el resto de 5 o más (López et al., 2013).

Por otro lado, un estudio realizado en Países Bajos por Vonk et al (2015), reporto que el $35 \%$ de los pacientes colostomizados consideraron tener una calidad de vida alta, 50\% moderada y el $15 \%$ refiere tener calidad de vida baja. A pesar que la calidad de vida del paciente colostomizado es evaluado de forma global en la anterior investigación, los datos emitidos se asemejan en gran medida al trabajo de investigación, debido a que la ubicación del estoma no es el único factor para determinar la calidad de vida del paciente colostomizado, sino también el sexo, la edad, el nivel de estudios, y el tiempo de permanencia del estoma, (Muñoz et al., 2011).

Por otro lado, en cuanto al nivel de la calidad de vida de los pacientes portadores de colostomía, como resultado se encontró que el 51,5\% presenta una calidad de vida entre media y baja, este estudio difiere con el estudio desarrollado en México por Hernández (2011) quien encontró que $14,92 \%$ de pacientes colostomizados presentaron una calidad de vida baja, esta diferencia considerable se explica porque el Estado mexicano en el marco de su política de salud contempla los derechos del paciente ostomizado y dentro de sus lineamientos está que el paciente debe recibir información desde el preoperatorio, así como los aspectos esenciales que conlleva vivir con un estoma, asimismo, existen auditorias sobre el buen desempeño del profesional a cargo de los pacientes colostomizados, siendo que ello deberían presentar 
Tipos de colostomía según su localización y la calidad de vida en los pacientes colostomizados que asisten al Consultorio Externo de Cirugía General del Hospital Nacional Guillermo Almenara Irigoyen, Lima, 2015

una calidad de vida adecuada (Herrera ,2012), en tanto en el Estado peruano, existe un modelo de atención integral de salud (MAIS) que atiende según etapas de vida , su función es de brindar promoción y prevención de enfermedades, sin embargo no se cuenta con un modelo de atención específica para enfermedades prevalentes, que tras una atención quirúrgica puede traer consigo alteraciones físicas, psicológicas y sociales así mismo, la demanda de pacientes y el poco recurso de materiales dificultan el desarrollo de la actividades del profesional de enfermería por ende en nuestro medio se reporta que la calidad de vida es alta en un menor porcentaje $(13,4 \%)$ en comparación con otros estudios en otros países.

También el estudio contempló la relación entre el tiempo de uso de la colostomía y la calidad de vida, se obtuvo como resultado un p-value $=0.036$ lo cual indica la relación de ambas variables, esta asociación se explica a que la recuperación de los pacientes, aumenta con el transcurso del tiempo así mismo la adaptabilidad humana, por ende la calidad de vida de un paciente colostomizado será medida por el tiempo y permanencia del estoma digestivo, a mayor tiempo, su calidad de vida mejorará positivamente (Pita, 2015), así mismo, Xiuxiu et al (2014) ejecutaron una investigación, cuyo objetivo fue observar la calidad de vida en pacientes con cáncer rectal con colostomía permanente en Xi'an- China en diferentes períodos después de la operación, encontrándose una buena calidad de vida en el primer mes posoperatorio en $28.70 \%$, tercer mes $54.42 \%$ y sexto mes $74.21 \%$.

La similitud de resultados se debe que los pacientes portadores de un estoma con menos de un año presentan menor actividad social y desarrollo personal, por otro lado los pacientes con más de un año manifiestan niveles de aceptación de su condición y manejan mejor el cambio de su imagen corporal y física, (Casado, 2012). Como resultado de la investigación presentada, se concluye que existe una relación significativa entre los tipos de colostomía según su localización y la calidad de vida de los pacientes colostomizados que asisten a consultorio externo de Cirugía General del Hospital General Guillermo Almenara Irigoyen.

\section{Declaración de financiamiento y de conflicto de intereses:}

El estudio fue financiado por los autores, quienes declaran no tener algún tipo de conflicto de interés en la investigación realizada.

\section{Correspondencia:}

Jairo Bastidas Chuquillanqui

Licenciado en Enfermería. Clínica Angloamericana. Alfredo Salazar Nº 350, San Isidro, Lima 27, Perú e-mail: jairo.bastidas@upeu.edu.pe

\section{REFERENCIAS BIBLIOGRÁFICAS}

Ardila, R. (2003). Calidad de vida: Una definición integradora. Revista Latinoamericana de Psicología, 35(2), 161-164.

Brito, J., Jiménez, K., Tolorza, G., Siqués, P., Rojas, F., \& Barrios, L. (2007). Impacto de la ostomía en el paciente y su entorno. Revista Chilena de Cirugía, 56(1), 31-34.

Castro, M., Castro, C., \& Clemente, H. (2013). Los cuidados de enfermería en el impacto psicológico del paciente. Ene. Revista de Enfermeria, 7(3).

Casado, C. (2012). Plan de cuidados. Cáncer de recto: paciente colostomizado. Rev. Reduca (Enfermería, Fisioterapia y Podología), 4 (1) 563-596. Recuperado de http://www. revistareduca.es/index.php/reduca-enfermeria/article/ viewFile/1009/1021

Castañeda, O. C., Garduño, M. A. C., Castillo, L. P., Bautista, M. D. P. S., Estévez, J. M. E., Espinosa, J. G. R., \& Corral, S. H. (2009). Epidemiología de ostomías de eliminación en diferentes unidades de salud del Distrito Federal. Revista CONAMED, 14(1), 15-19.
Cesaretti, I. U. R., Santos, V. L. C. G., \& Vianna, L. A. C. (2010). Qualidade de vida de pessoas colostomizadas com e sem uso de métodos de controle intestinal. Revista Brasileira de Enfermagem, 63(1), 16-21. http://doi. org/10.1590/S0034-71672010000100003.

Crespo, L. (2012). Plan de cuidados de enfermería estandarizados en el paciente ciolostomizado. Recuperado de http://repositorio.unican.es/xmlui/ bitstream/handle/10902/3940/CrespoVillazanL. pdf?sequence $=1$.

Carrasco, S. (2005). Metodología de la investigación científica, pautas metodológicas para diseñar y elaborar el proyecto de investigación. Lima-Perú: San Marcos.

Charúa-Guindic, L., Benavides-león, C. J., Villanuevaherrero, J. A., Jiménez-bobadilla, B., Abdo-francis, J. M., \& Hernández-labra, E. (2011). Calidad de vida del paciente ostomizado. Cirugía Digestiva, 79, 149-155.

Costa, A., \& Parrelli, M. (2011). La sexualidad del paciente con ostomía: revisión integradora. Revista de Enfermagem, 7, 4935-4943. http://doi.org/10.5205/ reuol.4700-39563-1-ED.0707esp201315. 
Ferreira-Umpiérrez, A. H. (2013). Viviendo con una ostomía: Percepciones y expectativas desde la fenomenología social. Texto E Contexto Enfermagem, 22(3), 687-694. http://doi.org/10.1590/S0104-07072013000300015.

Gonzalo, m. (2009). percepcion de la calidad de vida en el paciente colostomizado permanentemente. Journal of Coloproctology, 3(2), 70-75.

Hernández, F. (2011). Impacto de un plan de intervención educativa en la calidad de vida del paciente ostomizado. Rev. Reduca, 3 (2), 504-533. Recuperado de http://www. revistareduca.es/index.php/reduca-enfermeria/article/ viewFile/764/780.

Hernández, R., Fernández, C., \& Baptista, M. (2010). Metodología de la investigación. México: McGraw-Hill Interamericana.

Espinoza Herrera, M. (2003). Conocimientos y prácticas sobre el autocuidado que tienen los pacientes colostomizados que asisten a consulta de enfermería del hospital nacional Edgardo Rebagliti Martins. (Tesis para optar la licenciatura en enfermería). Recuperado de http://sisbib.unmsm.edu.pe/bibvirtualdata/tesis/salud/ espinoza_hm/enpdf/t_completo.pdf

Kimura, C. A., Kamada, I., Guilhem, D., \& Fortes, R. C. (2013). Perception of sexual activities and the care process in ostomized women. Journal of Coloproctology, 33(3), 145-150. http://doi.org/10.1016/j.jcol.2013.05.004.
OMS (2015). Organización Mundial de la Salud. Estrategia mundia sobre la percepcion de la calidad de vida.

Pita, P. et al. (2015). Caso clínico de dermatitis periestomal y dehiscencia en una colostomía. Rev. Enferm Dermatol, 9(24). Recuperado de http://dialnet.unirioja.es/servlet/ articulo?codigo $=5185639$

Sanchez, H. \& Reyes, C. (2002). Metodología y diseño en la investigación científica. Lima-Perú: Editorial Universitaria. Universidad Ricardo Palma

Villajuan, E. (2006). calidad de vida de pacientes Ostomizado que asisten a consulta de Enfermería del Instituto Nacional de Enfermedades Neoplásicas. Revista Facultad Nacional de Salud Publica, 61(3). http://doi. org/10.4067/S0718-40262009000300008

Xiuxiu, Q. (2012). Quality of life in rectal cancer patients with permanent colostomy in Xi'an. African Health Sciences, 14(1) 28-36. Recuperado de http://dx.doi. org/10.4314/ahs.v14i1.6

Recibido: 24/02/2016 Aceptado: 04/04/2016 